\title{
Care of People with Epilepsy in Rural Medical Centre of Developing Countries with Limited Resources: During the COVID-19 Pandemic Verma $A,{ }^{1}$ Kumar $A^{2}$
}

\author{
${ }^{1}$ Department of Neurology, \\ ${ }^{2}$ Department of Forensic Medicine and Toxicology \\ UP university of Medical Sciences, Saifai, \\ Etawah-206301 (U.P.), India. \\ Corresponding Author \\ Archana Verma \\ Department of Neurology, \\ Department of Forensic Medicine and Toxicology \\ UP university of Medical Sciences, Saifai, \\ Etawah-206301 (U.P.), India. \\ E-mail: archanashiva2010@rediffmail.com
}

\begin{abstract}
Coronavirus disease 2019 (COVID-19) has become a pandemic disease globally. This extraordinary situation is posing an enormous burden on the healthcare systems worldwide and is reshaping the way in which chronic disorders are managed. Pandemic has made epilepsy care even more demanding in rural part of developing countries. We outline the adverse circumstances in epilepsy care induced by the pandemic; propose emergency management and follow up care of people with epilepsy. There is a requirement for public health systems in resource poor countries to improve awareness, implement proper strategies of triage, acute treatment, telemedicine services and virtual check-ins.
\end{abstract}

\section{KEY WORDS}

COVID-19, Disease control, People with epilepsy, Self-management

\section{Citation}

Verma A, Kumar A. Care of People with Epilepsy in Rural Medical Centre of Developing Countries with Limited Resources: During the COVID-19 Pandemic. Kathmandu Univ Med J. 2020;COVID-19 Special Issue 70(2):99-101.

\section{INTRODUCTION}

Currently the whole world is confronting a pandemic of coronavirus disease 2019 (COVID-19) caused by severe acute respiratory syndrome coronavirus 2 (SARS-CoV-2). This new virus and disease were unidentified before the outbreak began in Wuhan, China, in December 2019. ${ }^{1}$ Globally as well as in India, COVID 19 has been a cause of concern and disruption. These are unusual times when focus of health system is to take care of patients suffering from COVID -19 as well as community, resulting in disruption of care of chronic disorders and needs reorientation. The first case of COVID- 19 in India was reported on $30^{\text {th }}$ January, 2020 and the total number of coronavirus cases in India jumped to 650,431 on $4^{\text {th }}$ July $2020 .^{2}$

The COVID-19 pandemic has overpowered health care frameworks in developed countries. It isn't amazing that the health impact of the COVID-19 pandemic sweeping the world has created multiple challenges for epilepsy care in India, which are very much distinct from those faced by the developed world. It's going to be far more terrible in resources poor countries where medical resources are scarce with the limited COVID-19 testing facilities, shortages of health care personnel, life saving instruments (ventilator etc) and personal protective equipments (PPE).

Around the world, it is assessed that nearly 50 million individuals are suffering from epilepsy and among them about $90 \%$ are from developing nations. ${ }^{3}$ There are in excess of 12 million persons with epilepsy (PWE) in India, contributing to almost one-sixth of the global burden. ${ }^{4}$ The frightening fact in rustic and underserved areas is that the prevalence of epilepsy is twice where health facilities are already sorely lacking. ${ }^{5}$ PWE is already much prone to co morbidities and stigma that negatively impact their quality of life.

Therefore, a new robust framework is needed that keep away from avoidable transportation while expanding the availability of medical resources according to demand. 
Here we review approaches that provide best possible care to people with epilepsy (PWE) with limited resource during a pandemic event like COVID-19 and raise a conceptual management structure with a special focus on telemedicine.

\section{Challenges faced in resource poor countries during pandemic}

All through COVID-19 pandemic management of epilepsy in the rustic parts of resource poor countries are particularly challenging as there is poor health care infrastructure, shortage of trained professionals, poor transport facilities, lower socioeconomical status, low illiteracy rate, non accessibility of medications, fear of contracting infection and non adherence to antiepileptic drugs (AEDs). Online pharmacies and telemedicine services are also seldom available in these areas.

\section{Emergency management of epilepsy during the pandemic}

The organization of neurological emergencies and admissions is particularly demanding at a time when a high number of patients are infected with the virus. Emergency care of PWE suspected of having COVID-19 is of special concern. As per the Ministry of Health and Family Welfare (MoHFW) Government of India guidelines, these cases are supposed to go to assigned COVID -19 care government hospitals most of them are in districts and at state hospitals. ${ }^{2}$

Before directly visiting a hospital; a clear medical history is critical to the diagnosis, telemedicine consultation with video footage or online consulting with community caregivers or with neurologist is recommended. By examining these home-based video recordings one can get better diagnostic accuracy, helping in the differential diagnosis with other nonepileptic paroxysmal disorders or in the classification of epileptic seizures. ${ }^{6}$ This approach could avoid blind seeking for medical support and ease the psychological impact from first seizure.

Evaluation and urgent care by a healthcare professional are always obligatory and also during the COVID-19 pandemic in patients with a status epilepticus (including nonconvulsive status epilepticus), first-ever seizure, if seizures are followed by unusually prolonged postictal confusional symptoms, impaired vital parameters after the seizure(s), seizure-related injury, or convulsive seizure(s) occurring in subjects with diabetes, heart disease, or pregnancy. ${ }^{7,8}$

Detail screening history should be taken prior to emergency admission. Health care workers Patients and their companions should be asked for typical symptoms of COVID-19, such as fever, sore throat, difficulty in respiration etc, within 14 days of exposure or a contact history with suspected or confirmed cases or living in hot spot. Patients' temperature should be taken prior to consultation, and individuals presenting with fever should be first referred to flu outpatient door and all safety protocols should be followed.

Ensure meticulous COVID-19 screening and triage protocols in the emergency department. Neurological emergency (including consultation, CT/MRI, and electrophysiology rooms) should be strictly separated from fever clinics to avoid direct interaction with febrile patients. For the emergency management different units and subspecialties of neurology department should be merged into a single unit in centre with significant shortage of neurologist and residents. In some institute, non-overlapping teams were created to minimize transmission among healthcare workers. In this protocol, each department is covered by 2 non -overlapping teams (rotating in 3-day cycles: 3 days on and 3 days off), in which members have contact only within the same team. ${ }^{9}$ All staff (research faculty, research fellows, and students) not required for clinical duties should remain at home.

For each patient only one family attendant is allowed, and that to must have had the same screening tests to exclude COVID-19 before proceeding to entering the ward. Temperature screening of anyone entering the ward must be done by a thermal scanner and everyone in the ward should monitor their body temperature regularly. Medical staff in general wards should wear disposable work caps, medical protective masks and work clothes during the pandemic period or working in areas with high risk of COVID-19. A robust staff awareness training program must be conducted for all staff to ensure personal hygiene and to minimize any spreading of infection.

\section{Care of people with epilepsy}

Most important actions taken by government aimed at slowing the spread of the viral infection by lockdown, social distancing and self-isolation to minimizing contacts between potentially infected individuals and healthy subjects. Nevertheless, all these measures complicate the management of PWE by causing or worsening concomitant anxiety and depression, hampering their contacts with physicians, psychologist and leading to shortages or erratic supply of AEDs.

To address these difficulties, some approaches must be considered, including expanded telemedicine coverage, these services can prove extremely helpful in supporting remote healthcare in PWE during the current COVID-19 pandemic, mostly for their role in facilitating the interactive exchange of information between patients and physicians. ${ }^{10}$ Telemedicine isn't sub-par compared to customary face-toface visits with regard to seizure control, hospitalizations, emergency room visits, or AEDs adherence. ${ }^{11,12}$

Self-management, these aptitudes incorporate management of both medical and nonmedical matters for example emotion or role changing. ${ }^{13}$ Utilizing telephone application-based communications (SMS, Whatsapp), video 
recording of the ictal event, maximizing the accessibility of AEDs by all means, obtain a repeat prescription and encouraging for drug adherence. The government leaders are relied upon to ensure regular and uninterrupted availability of AEDs within the sight of lockdown.

PWE must be reassured that in the absence of risk factors, including older age, immobility, coexistent respiratory disease, diabetes mellitus, hypertension, severe heart disease, or on immunosuppressant, are not at a higher risk for COVID-19 infections.

People with epilepsy and their families/caregivers must have a clear perceptive of when and how to use rescue medications (e.g., benzodiazepines via buccal, nasal, or rectal routes or oral if they can be safely swallowed), when to repeat and when to visit emergency department.14 All these measures will keep PWE away from repetitive appointment to the hospital, lowering the risk of exposure to the pandemic.

Strong mental and emotional health support is another aspect of importance. ${ }^{15}$ due to the fact that an estimate of $30-50 \%$ of PWEs suffers from anxiety even without an epidemic. ${ }^{16-17}$ Lifestyle issues ought to be reinforced: the need for regular sleep, consistency in current routines, healthy eating, exercise and avoidance of alcohol.

\section{REFERENCES}

1. WHO; Coronavirus disease (COVID-2019) situation reports 2020 https://www.who.int/emergencies/ diseases/novelcoronavirus-2019/ situation-reports

2. Official Updates Coronavirus - COVID-19 in India - mygov.in https:// www.mohfw.gov.in/

3. GBD 2016 Epilepsy Collaborators. Global, regional, and national burden of epilepsy, 1990-2016: a systematic analysis for the Global Burden of Disease Study 2016. Lancet Neurol. 2019;18:357-75.

4. WHO: World Health Organization (WHO) Factsheet: Epilepsy 2015 (cited May 1, 2015). http://www.who.int/mediacentre/factsheets/ fs $999 /$ en/.

5. Ngugi AK, Kariuki SM, Bottomley C, Kleinschmidt I, Sander JW, Newton CR: Incidence of epilepsy: a systematic review and metaanalysis. Neurology. 2011; 77: 1005-12.

6. Tatum WO, Hirsch LJ, Gelfand MA, Acton EK, LaFrance JrWC, Duckrow $\mathrm{RB}$, et al. Assessment of the predictive value of outpatient smartphone videos for diagnosis of epileptic seizures. JAMA Neurol. 2020. https:// doi.org/10.1001/jamaneurol.2019. 4785.

7. National Center for Chronic Disease Prevention and Health Promotion (NCCDPHP). Seizure first aid. Available online at: . https://www.cdc. gov/epilepsy/about/firstaid. htm; 2019. [Accessed 30 April 2020].

8. Kinney MO, Brigo F, Kaplan PW. Optimizing status epilepticus care during the COVID-19 pandemic. Epilepsy Behav. 2020. https://doi. org/10.1016/j.yebeh.2020. 107124.

9. Ozoner B, Gungor A, Hasanov T, et al. Neurosurgical Practice During Coronavirus Disease 2019 (COVID-19) Pandemic. World Neurosurgery. Volume 140, August 2020, Pages 198-207
Yoga is acknowledged to bring stress reduction and relaxation. The effect of yoga on the EEG and the autonomic nervous system has been reported. There are different types of yoga involving postural exercises (asanas), breath control (pranayama) and meditation. Yoga practices in PWE presents an economical, non-invasive tool and it also complements to epilepsy control and improvement in quality of life. ${ }^{18}$ Psychologist consultations on telemedicine are important to reduce stress and anxiety.

\section{CONCLUSION}

Diverse aspect of epilepsy treatment needs to be reformed to provide optimized services during such pandemic. There is a requirement for public health systems in rural part of resources poor countries to improve epilepsy awareness and to implement proper strategies of triage, acute treatment, epilepsy care, telemedicine services. Maximize utilization of constrained assets' may help health care professionals to provide optimal care to PWE during the pandemic. All these measures will help to maintain the continuum of care and reduce morbidity and mortality in PWE.

10. Kissani N, Lengané YTM, Patterson V, Mesraoua B, Dawn E, Ozkara C, et al. Telemedicine in epilepsy: how can we improve care, teaching, and awareness? Epilepsy Behav. 2020;103:106854.

11. Bahrani K, Singh MB, Bhatia R, Prasad K, Vibha D, Shukla G, et al. Telephonic review for outpatients with epilepsy-a prospective randomized, parallel group study. Seizure. 2017;53:55-61.

12. Reider-Demer M, Raja P, Martin N, Schwinger M, Babayan D. Prospective and retrospective study of videoconference telemedicine follow-up after elective neurosurgery: results of a pilot program. Neurosurg Rev. 2018;41:497-501.

13. Barlow J, Wright C, Sheasby J, Turner A, Hainsworth J. Selfmanagement approaches for people with chronic conditions: a review. Patient Educ Couns. 2002;48(2):177-87

14. French JA, Brodie JM, Caraballo R, et al. Keeping People with Epilepsy Safe During the COVID-19 Pandemic. Neurology. 2020 Jun 9;94(23):1032-7

15. Xiang YT, Yang Y, Li W, Zhang L, Zhang Q, Cheung T, et al. Timely mental health care for the 2019 novel coronavirus outbreak is urgently needed. Lancet Psychiatry. 2020;7(3):228-9.

16. Munger Clary HM, Snively BM, Hamberger MJ. Anxiety is common and independently associated with clinical features of epilepsy. Epilepsy Behav. 2018;85:64-71.

17. Jones JE, Bell B, Fine J, Rutecki P, Seidenberg M, Hermann B. A controlled prospective investigation of psychiatric comorbidity in temporal lobe epilepsy. Epilepsia. 2007;48(12):2357-60.

18. Lundgren T, Dahl J, Yardi N, Melin L. Acceptance and Commitment Therapy and yoga for drug-refractory epilepsy: A randomized controlled trial. Epilepsy Behav. 2008;13:102-8. 